\title{
Euler-Lagrange Equations of Networks with Higher-Order Elements
}

\author{
Zdeněk BIOLEK ${ }^{1,2}$, Dalibor BIOLEK ${ }^{1,2}$ \\ ${ }^{1}$ Dept. of Microelectronics, Brno University of Technology, Technická 10, 61600 Brno, Czech Republic \\ ${ }^{2}$ Dept. of Electrical Engineering, University of Defense, Kounicova 65, 66210 Brno, Czech Republic
}

zdenek.biolek@gmail.com, dalibor.biolek@unob.cz

Submitted April 15, 2017 / Accepted April 15, 2017

\begin{abstract}
The paper suggests a generalization of the classic Euler-Lagrange equation for circuits compounded of arbitrary elements from Chua's periodic table. Newly defined potential functions for general $(\alpha, \beta)$ elements are used for the construction of generalized Lagrangians and generalized dissipative functions. Also procedures of drawing the Euler-Lagrange equations are demonstrated.
\end{abstract}

\section{Keywords}

Periodic table of fundamental elements, higher-order elements, content, energy, action, evolution, Lagrangian, dissipation function, FDNR, inerter

\section{Introduction}

Euler-Lagrange equations of motion are a well-established part of physics with its potential application in mechanics, electrical engineering, and various multi-disciplinary branches. They express the equilibrium of generalized forces or fluxes, which provides the energy-balance between energy dissipation and storage. In each of the fields of science, we can find adequate forms of the resistor as a dissipative element, and the capacitor and inductor that represent the accumulation and inertial elements for energy storing [1]. The transition from the energetic description to the equation of motion is implemented by a systematic differentiation of dissipative function of the system and its Lagrangian with respect to system coordinates and time. The classic form of these equations for balancing generalized vector forces $\boldsymbol{v}$ (whose electrical analogy can be voltages $\boldsymbol{v}$ ) in a system given by generalized coordinates $\boldsymbol{q}$ (often acting as electrical charges $\boldsymbol{q}$ ) is

$$
\frac{\mathrm{d}}{\mathrm{d} t}\left(\frac{\partial L}{\partial \dot{\boldsymbol{q}}}\right)-\frac{\partial L}{\partial \boldsymbol{q}}+\frac{\partial C}{\partial \dot{\boldsymbol{q}}}=\boldsymbol{v}_{\text {ext }}
$$

where the Lagrangian $L=T^{*}-V$, formed as the difference between kinetic co-energy and potential energy, describes the state of reactive elements, i.e. inductors and capacitors, and the dissipative function $C$ called content illustrates a dissipation of energy on lossy elements, i.e. resistors; $\boldsymbol{v}_{\mathrm{ext}}$ is the vector of external excitation. Equation (1) can be read from left to right as follows: voltages at inductors as derivatives of magnetic fluxes with respect to time, plus voltages at capacitors, plus voltages at resistors within the loop are equal to the voltages of voltage sources in the loop.

The current form of the Euler-Lagrange equations is no longer sufficient for describing systems that contain, in addition to resistors, capacitors, and inductors, also other fundamental elements.

The theoretical foundations of these elements were laid by L. Chua in several successive steps in the seventies and eighties of the 20th century. In [2], he introduced the memristor into the circuit theory, and in [3] he pointed out the existing regularities among fundamental electrical elements organized in the so-called periodic table of HigherOrder Elements (HOEs) shown in Fig. 1.

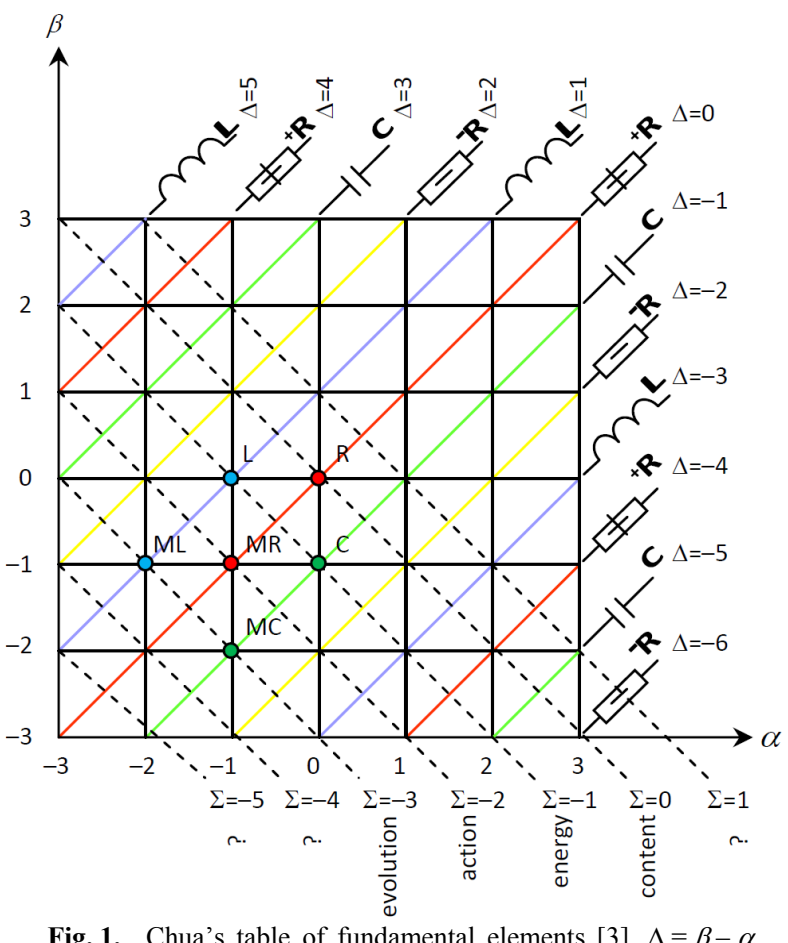

Fig. 1. Chua's table of fundamental elements [3]. $\Delta=\beta-\alpha$, $\Sigma=\alpha+\beta$ 
Chua's table of elements, analogous to Mendeleev's periodic table of chemical elements, shows that the existence of all currently known fundamental electrical elements (resistor $\mathrm{R}$, capacitor $\mathrm{C}$, inductor $\mathrm{L}$, memristor MR, meminductor $\mathrm{ML}$, and memcapacitor $\mathrm{MC}$ ) is a logical consequence of a symmetry that exists between $v^{(\alpha)}$ and $i^{(\beta)}$ variables, where positive integers $\alpha$ and $\beta$ stand for the respective orders of derivatives of terminal voltage $v$ and current $i$ with respect to time, negative integers mean the orders of time-domain integrals, and zeros refer to the voltage $v$ and current $i$ itself. By using these symbols, we can denote the charge as $q=i^{(-1)}$, flux $\varphi=v^{(-1)}$, and their time-domain integrals as $\sigma=i^{(-2)}$ and $\rho=v^{(-2)}$. This means that the resistor with the $v-i$ constitutive relation (CR) occupies the position $(0,0)$; similarly, the capacitor, inductor, and memristor coordinates are $(0,-1),(-1,0)$, and $(-1,-1)$, and the indices of the unconventional elements such as the memcapacitor and meminductor are $(-1,-2)$ and $(-2,-1)$. The position of the above six elements is designated in Fig. 1.

The classic paper [3] from 1980 reveals a specific attribute of the elements occupying the $\Delta$-diagonals of Chua's table ${ }^{1}$. The small-signal models of the elements of each $\Delta$-diagonal have always the same character, either of the resistive, capacitive, or inductive type. From the smallsignal point of view, there are only frequency-dependent positive and negative resistors ${ }^{+} \mathrm{R}$ and ${ }^{-} \mathrm{R}$, capacitors $\mathrm{C}$, and inductors $\mathrm{L}$ of the order $\Delta$. In Fig. 1, the corresponding types of the $\Delta$-diagonals are colored (in red, violet, yellow, and green) and labeled by the symbol of the element.

Since the elements placed together on one of the $\Sigma$-diagonals have a fixed sum of indices $\Sigma=\alpha+\beta$, their potential functions will be of the same character and physical dimension. For example, the capacitor and inductor are located on the same $\Sigma$-diagonal $(\Sigma=-1)$, and their potential functions have the character of energy with the dimension of Joule $[\mathrm{J}]$. The memcapacitor and meminductor on a different $\Sigma$-diagonal $(\Sigma=-3)$ have the potential functions called the evolution, with the physical dimension of [ $\left.\mathrm{Js}^{2}\right]$.

The first references on how to construct Euler-Lagrange equations for circuits with memristors, memcapacitors and meminductors are in [4] (Cohen) and [5] (Jeltsema). The paper [5] is based on the crucial idea that the potential functions, the Euler-Lagrange equations being compounded of them, need to be assembled from the constitutive relations (CRs) of the elements, exactly as it was for the $\mathrm{R}, \mathrm{C}$, and $\mathrm{L}$ elements. The importance of this idea will be demonstrated on the examples of the resistor and memristor. The CRs of all six hitherto identified fundamental elements together with their symbols are presented in Fig. 2.

1 The diagonals of Chua's table with fixed difference $\Delta=\beta-\alpha$ (sum $\Sigma=\alpha+\beta)$ will be denoted hereafter as $\Delta$-diagonals ( $\Sigma$-diagonals).

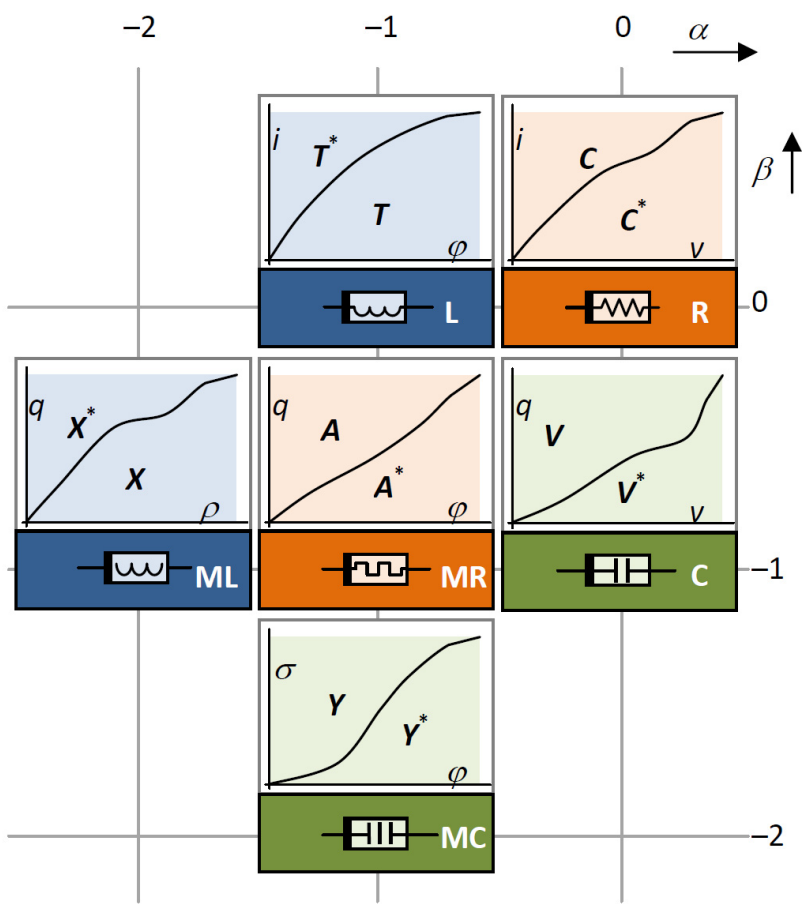

Fig. 2. Potential (co)functions of six fundamental elements (co-functions are marked with an asterisk) as filling areas of the CRs: content $C$, kinetic and potential energy $T$ and $V$, action $A$, kinetic and potential evolution $X$ and $Y$ [6].

The dissipative function $C$ (content) corresponds to the area under the $v-i$ constitutive relation of the resistor. The content is therefore obtained via integrating the CR with respect to the current, while the resistor voltage can be obtained via differentiating the content with respect to current (see the last left-hand term in (1)). However, this procedure will fail for the memristor: the integral of the $v-i$ characteristic with respect to current depends not only on the instantaneous current, but also on the value of the charge passed through, i.e. on the course of the path of integration. The content is no longer a potential function, thus the memristor voltage is not given by the gradient of content, and the recipe (1) for finding the voltage does not work anymore. This is the reason why in [5] Jeltsema proposes to start from the $\varphi-q \mathrm{CR}$ of the memristor, and from the area under this characteristic that corresponds to the potential function of the memristor called action (see Fig. 2). The gradient of the action with respect to charge is then equal to the flux $\varphi$. An analogous procedure is also applied to memcapacitor and meminductor. The original Equation (1) of the equilibrium of the loop voltages is replaced by the flux balance

$$
\frac{\mathrm{d}}{\mathrm{d} t}\left(\frac{\partial L_{\mathrm{M}}}{\partial \dot{\boldsymbol{\sigma}}}\right)-\frac{\partial L_{\mathrm{M}}}{\partial \boldsymbol{\sigma}}+\frac{\partial A}{\partial \dot{\boldsymbol{\sigma}}}=\boldsymbol{\varphi}_{\text {ext }}
$$

where the Lagrangian $L_{\mathrm{M}}=X^{*}-Y$ is formed from the areas under the CRs of meminductors and memcapacitors, $A$ is the memristive potential called action, and $\varphi_{\mathrm{ext}}$ is the timedomain integral of the external driving voltage. Equation (2) can be read from left to right as follows: The meminductor magnetic fluxes, plus the memcapacitor fluxes, plus 
memristor fluxes within closed loop are equal to the flux supplied by the sources in the loop.

The rule (2) enables constructing the equations of motion just on the knowledge of the CRs of the circuit elements. As a limitation, the circuit cannot include a combination of the mem-elements and non-linear elements without memory. We have resolved this issue in [6]. The Euler-Lagrange equations of circuits consisting of any combinations of non-linear resistors, capacitors, inductors, and their memory variants, have the form of

$$
\begin{gathered}
\frac{\mathrm{d}}{\mathrm{d} t}\left(\frac{\mathrm{d}}{\mathrm{d} t}\left(\frac{\partial L_{M}}{\partial \dot{\boldsymbol{\sigma}}}\right)-\frac{\partial L_{M}}{\partial \boldsymbol{\sigma}}+\frac{\partial A}{\partial \dot{\boldsymbol{\sigma}}}\right)+ \\
\frac{\mathrm{d}}{\mathrm{d} t}\left(\frac{\partial L}{\partial \ddot{\boldsymbol{\sigma}}}\right)-\frac{\partial L}{\partial \dot{\boldsymbol{\sigma}}}+\frac{\partial C}{\partial \ddot{\boldsymbol{\sigma}}}=\boldsymbol{v}_{\text {ext }} .
\end{gathered}
$$

Equation (3) represents a generalization of classic Euler-Lagrange equation (1) for circuits containing not only the non-linear R, C, and L elements, but also the mem-elements.

The main focus of the paper is on generalizing (1) for circuits with arbitrary combinations of arbitrary elements from Chua's table. The paper structure is as follows. The general form of the equation of motion for circuits made up of arbitrary elements from Chua's table is derived in Sec. 2, starting from the potential functions of the respective elements. In Sec. 3, this equation is identified in a form that is compatible with the classic form (1), i.e. it is expressed via the generalized Lagrangians and the generalized dissipative potentials. In Sec. 4, the mathematical apparatus obtained is applied to a specific frequency filter containing FDNRs (Frequency-Dependent Negative Resistors), which can be considered as small-signal versions of the $(1,-1)$ element. The last section deals with the construction of equations for a vibration absorber employing the mechanical element $(1,0)$, also known as the inerter.

\section{Equation of Motion for Circuits with Higher-Order Elements}

Consider a general two-terminal element $\varepsilon=(\alpha, \beta)$ with the terminal voltage $v(t)$ and current $i(t)$. According to the convention introduced in [3], consider the directions of voltage and current as shown in Fig. 3(a).

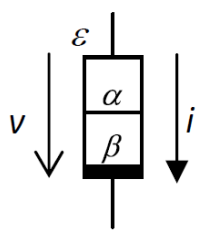

(a)

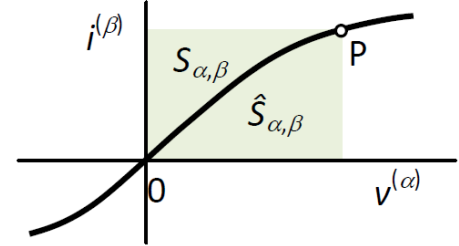

(b)
Fig. 3. (a) Symbol of the element $\varepsilon=(\alpha, \beta)$ and the orientation of its voltage and current, (b) an example of CR of the element and the geometrical meaning of the corresponding potential functions
The two-terminal element preserves, under any circumstances, an unambiguous constitutive relation between $v^{(\alpha)}$ and $i^{(\beta)}$. Then the integrals

$$
S_{\alpha, \beta}=\int v^{(\alpha)} \mathrm{d} i^{(\beta)}, \hat{S}_{\alpha, \beta}=\int i^{(\beta)} \mathrm{d} v^{(\alpha)}
$$

correspond to the relevant filling areas between the CR curve and the axes (the areas are related to the current operating point $\mathrm{P}$ in Fig. 3(b)) and they behave as potential functions: their gradients are again the quantities $v^{(\alpha)}$ and $i^{(\beta)}$ :

$$
v^{(\alpha)}=\frac{\mathrm{d} S_{\alpha, \beta}}{\mathrm{d} i^{(\beta)}}, i^{(\beta)}=\frac{\mathrm{d} \hat{S}_{\alpha, \beta}}{\mathrm{d} v^{(\alpha)}} .
$$

From comparing Fig. 2 and Fig. 3(b) it follows that the potential functions of six hitherto identified fundamental elements are special cases of general potential functions (4), e.g. content $C=S_{0,0}$, kinetic energy $T=\hat{S}_{-1,0}$, and potential co-evolution $Y=\hat{S}_{-1,-2}$.

Equation (5) will be used for constructing the equations of motion; the elements used therein being represented by their potential functions. For the loop in Fig. 4(a) or for the node in Fig. 4(b), it holds for any integers $a$ and $b$

$$
\sum_{j} v_{j}^{(a)}=v_{\mathrm{ext}}^{(a)}, \quad \sum_{k} i_{k}^{(b)}=i_{\mathrm{ext}}^{(b)} .
$$

For $a=0$ and $b=0$, we get the classic form of both Kirchhoff's laws, i.e. Kirchhoff's Voltage Law (KVL) and Kirchhoff's Current Law (KCL). The case $a=-1$ and $b=-1$ leads to Kirchhoff's Flux Law (KFL) and Kirchhoff's Charge Law (KCHL) [6]. Equation (6) generally expresses the validity of Kirchhoff's Voltage ${ }^{(a)}$ Law and Kirchhoff's Current ${ }^{(b)}$ Law, i.e. $\mathrm{KV}^{(a)} \mathrm{L}$ and $\mathrm{KC}^{(b)} \mathrm{L}$.

For $a=0, b=0, \quad$ Equation (5) and elementary arrangements yield the following forms of KVL and KCL for the loop and the node in Fig. 4

$$
\sum_{j}\left(\frac{\mathrm{d} S_{\alpha_{j}, \beta_{j}}}{\mathrm{~d} i^{\left(\beta_{j}\right)}}\right)^{\left(-\alpha_{j}\right)}=v_{\mathrm{ext}}, \sum_{k}\left(\frac{\mathrm{d} \hat{S}_{\alpha_{k}, \beta_{k}}}{\mathrm{~d} v^{\left(\alpha_{k}\right)}}\right)^{\left(-\beta_{k}\right)}=i_{\mathrm{ext}}
$$

Consider generally non-linear CRs of all elements within a loop or connected to a node. Equations (7) are differential equations with respect to the variable $i^{\left(\beta_{\mathrm{min}}\right)}$ or

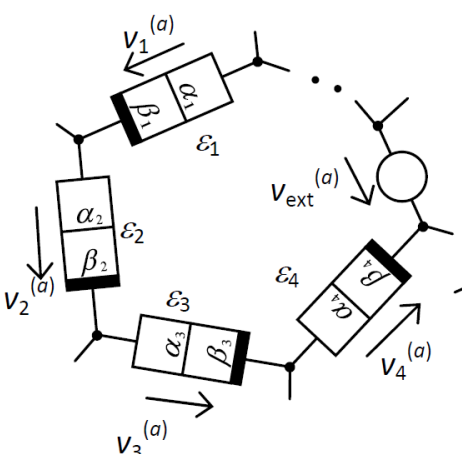

(a)

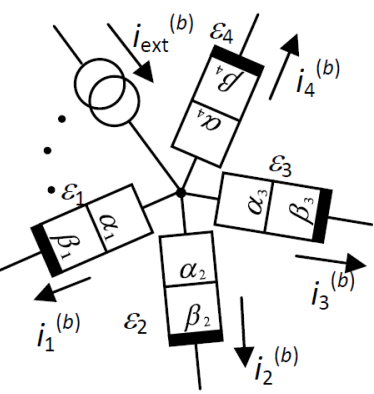

(b)
Fig. 4. (a) $\mathrm{KV}^{(a)} \mathrm{L}$ for a loop and (b) $\mathrm{KC}^{(b)} \mathrm{L}$ for a node. 
$v^{\left(\alpha_{\min }\right)}$, where $\beta_{\min }=\min \left\{\beta_{j}\right\}$ or $\alpha_{\min }=\min \left\{\alpha_{k}\right\}$, but only for elements with non-positive indices $\alpha_{j}$ or $\beta_{k}$. In other cases, Equations (7) are integral-differential or purely integral equations. If the equations of motion are required in the form of differential equations, we construct equations for $\mathrm{KV}^{(a)} \mathrm{L}$ and $\mathrm{KC}^{(b)} \mathrm{L}$, where $a=\max \left\{\alpha_{j}\right\}, b=\max \left\{\beta_{k}\right\}$. The final equations are

$$
\sum_{j} \frac{\mathrm{d}^{a-\alpha_{j}}}{\mathrm{~d} t^{a-\alpha_{j}}}\left(\frac{\mathrm{d} S_{\alpha_{j}, \beta_{j}}}{\mathrm{~d} i^{\left(\beta_{j}\right)}}\right)=v_{\text {ext }}^{(a)}, \sum_{k} \frac{\mathrm{d}^{b-\beta_{k}}}{\mathrm{~d} t^{b-\beta_{k}}}\left(\frac{\mathrm{d} \hat{S}_{\alpha_{k}, \beta_{k}}}{\mathrm{~d} v^{\left(\alpha_{k}\right)}}\right)=i_{\text {ext }}^{(b)}
$$

Note that the zero derivative of the function is equal to this function. It is clear that if the loop contains only resistors, capacitors, and inductors, then $a=0$, and the first equation in (8) transforms to (1).

\section{Generalization of Euler-Lagrange Equation}

General Equations (7) or (8) should be modified in order to resemble the form of (1). To do this, it is necessary to express the potential functions $S_{\alpha_{k}, \beta_{k}}$ and $\hat{S}_{\alpha_{k}, \beta_{k}}$, and also (1) using the language of functions, co-functions, Lagrangians, and generalized dissipative functions.

Today, three types of potential function and co-function are generally accepted: the energy and co-energy, introduced by Cherry [7] for capacitors and inductors; Millar's content and co-content for resistive elements [8]; and action and co-action, utilized by Chua for memristors [2]. The evolution and co-evolution for memcapacitors and meminductors are suggested in [6]. For the remaining elements from Chua's table, no potential functions and cofunctions have been hitherto established.

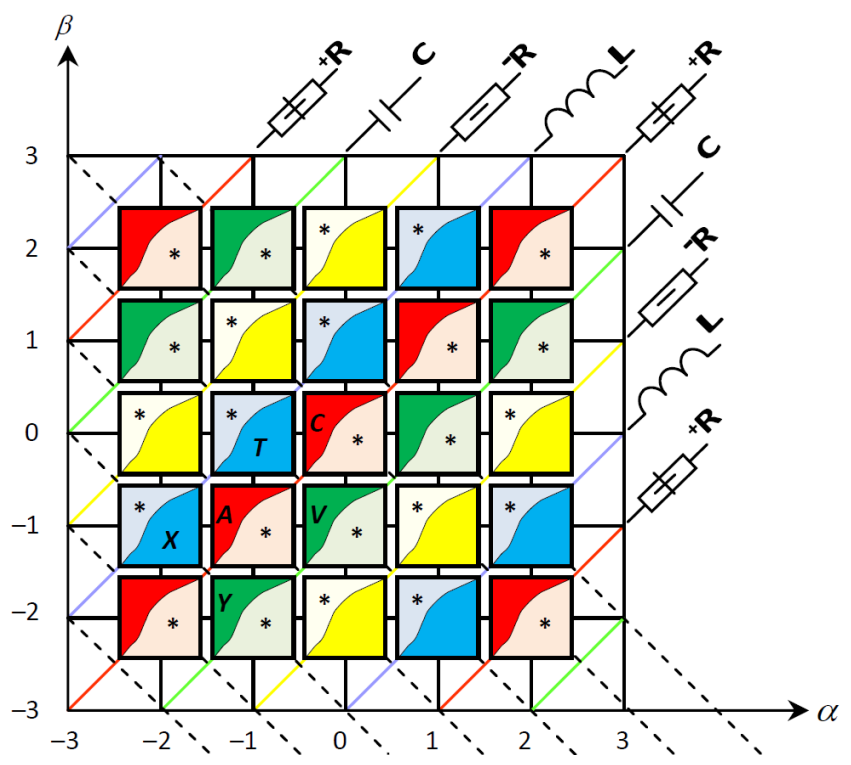

Fig. 5. The arrangement of potential functions and co-functions in Chua's table. The functions correspond to the colored areas in the CRs, the co-functions to areas denoted by * symbols.
The simplest possible rule for deciding which one from the pair $S_{\alpha, \beta}$ and $\hat{S}_{\alpha, \beta}$ is a function and which a cofunction can be deduced from Figs. 1, 2 and 3:

$$
S_{\alpha, \beta}=\left\{\begin{array}{l}
\text { function for } \varepsilon \in{ }^{+} \mathrm{R} \cap \mathrm{C} \\
\text { cofunction for } \varepsilon \in{ }^{-} \mathrm{R} \cap \mathrm{L}
\end{array} .\right.
$$

The rule for $\hat{S}_{\alpha, \beta}$ is quite opposite. The arrangement of the potential functions and co-functions across the table is shown in Fig. 5.

The elements of the same type, which share the $\Delta$-diagonal, have the same rule of distributing the potential functions and co-functions. This rule is always applied to two adjacent diagonals. The opposite rule holds for the subsequent or preceding two diagonals. For the $\Sigma$-diagonals, the rule alternates depending on switching from one to the other element.

Let us introduce a uniform notation for the potential functions and co-functions of a general $(\alpha, \beta)$ element as follows:

$$
\text { potential function }=\left\{\begin{array}{c}
{ }^{+} D_{\alpha, \beta} \text { for } \varepsilon \in{ }^{+} \mathrm{R}, \\
-D_{\alpha, \beta} \text { for } \varepsilon \in{ }^{-} \mathrm{R}, \\
V_{\alpha, \beta} \text { for } \varepsilon \in \mathrm{C}, \\
T_{\alpha, \beta} \text { for } \varepsilon \in \mathrm{L} .
\end{array}\right.
$$

The symbol ${ }^{*}$ should be added for co-functions. According to this notation, the potential energy of a capacitor is $V=V_{0,-1}$, and the potential evolution of a memcapacitor is $Y=V_{-1,-2}$.

For the potential functions (10), Equations (7) for the $\mathrm{KVL}$ or KCL are in the form

$$
\begin{aligned}
& \sum_{l}\left(\frac{\mathrm{d} T_{\alpha_{l}, \beta_{l}}^{*}}{\mathrm{~d} i^{\left(\beta_{l}\right)}}\right)^{\left(-\alpha_{l}\right)}+\sum_{c}\left(\frac{\mathrm{d} V_{\alpha_{c}, \beta_{c}}}{\mathrm{~d} i^{\left(\beta_{c}\right)}}\right)^{\left(-\alpha_{c}\right)}+ \\
& \sum_{r-}\left(\frac{\mathrm{d}^{-} D_{\alpha_{r-}, \beta_{r}}^{*}}{\mathrm{~d} i^{\left(\beta_{r-}\right)}}\right)^{\left(-\alpha_{r-}\right)}+\sum_{r+}\left(\frac{\mathrm{d}^{+} D_{\alpha_{r+}, \beta_{r+}}}{\mathrm{d} i^{\left(\beta_{r+}\right)}}\right)^{\left(-\alpha_{r+}\right)}=v_{\mathrm{ext}},
\end{aligned}
$$

or

$$
\begin{aligned}
& \sum_{l}\left(\frac{\mathrm{d} T_{\alpha_{l}, \beta_{l}}}{\mathrm{~d} v^{\left(\alpha_{l}\right)}}\right)^{\left(-\beta_{l}\right)}+\sum_{c}\left(\frac{\mathrm{d} V_{\alpha_{c}, \beta_{c}}^{*}}{\mathrm{~d} v^{\left(\alpha_{c}\right)}}\right)^{\left(-\beta_{c}\right)}+ \\
& \sum_{r-}\left(\frac{\mathrm{d}^{-} D_{\alpha_{r-}, \beta_{r-}}}{\mathrm{d} i^{\left(\alpha_{r-}\right)}}\right)^{\left(-\beta_{r-}\right)}+\sum_{r+}\left(\frac{\mathrm{d}^{+} D_{\alpha_{r+}, \beta_{r+}}^{*}}{\mathrm{~d} i^{\left(\alpha_{r+}\right)}}\right)^{\left(-\beta_{r+}\right)}=i_{\text {ext }}
\end{aligned}
$$

where $l, c, r^{+}$, and $r$ - are the indices of the elements of the type of $\mathrm{L}, \mathrm{C},{ }^{+} \mathrm{R}$ and ${ }^{-} \mathrm{R}$ connected in a loop or to a node.

According to Fig. 6, all three classical RCL elements, which constitute circuits complying with the Euler-Lagrange equations of type (1), are located in the highlighted stripe $\mathfrak{S}_{0}$ (under the number $n=0$ ), which is delimited by the $\Sigma$-diagonals for $\Sigma=-1$ and $\Sigma=0$. In this stripe, however, also other dissipative and reactive elements occur. 


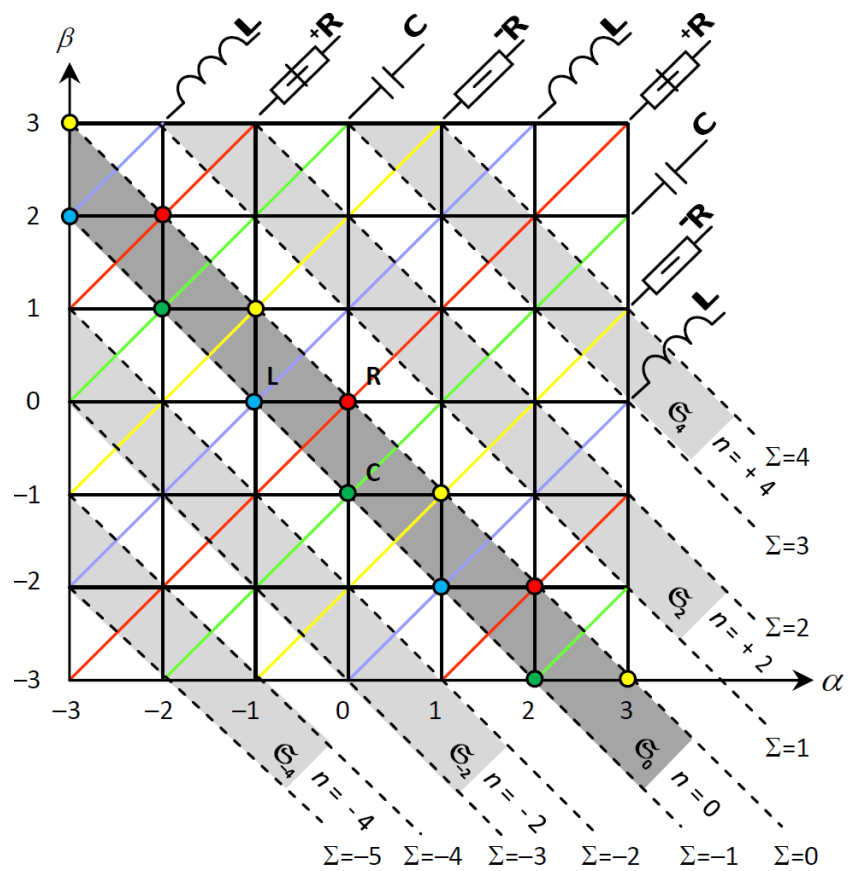

Fig. 6. Elements from the periodical table, sorted on the basis of belonging to various orders $n$ of the Lagrangian and dissipative function. In each stripe, the dissipative elements of positive (red) and negative (yellow) resistor, and reactive elements of inductive (blue) and capacitive (green) type alternate periodically.

From the small-signal point of view, there are the elements with positive and negative resistances ${ }^{+} \mathrm{R}$ and ${ }^{-} \mathrm{R}$, capacitances $\mathrm{C}$ and inductances L (in Fig. 6 they are marked with color circles). The potential functions of all dissipative elements on the diagonal $\Sigma=0$ have the physical dimension of power $\left[\mathrm{Js}^{-1}\right]$. Analogously, the potential functions of the reactive elements on the diagonal $\Sigma=-1$ are energies $[\mathrm{J}]$. Due to the identical physical dimensions of the potential functions and their possible additivity, the question arises whether we could construct an extended version of (1) for arbitrary elements from the $\mathfrak{S}_{0}$ stripe, in which the content $C$ would be replaced by a generalized dissipative function $D_{0}$ that would include all dissipative elements, and the Lagrangian $L$ would be replaced by a generalized Lagrangian $L_{0}$, which would cover all the reactive elements in the $\mathfrak{S}_{0}$ stripe.

This consideration also refers to a general stripe $\mathfrak{S}_{n}$. Let us introduce a dissipative function ${ }^{+} D_{n}$ for the elements of ${ }^{+} \mathrm{R}$ type and the dissipative function ${ }^{-} D_{n}$ for the elements of ${ }^{-} \mathrm{R}$ from the $\mathfrak{S}_{n}$ stripe:

$$
{ }^{+} D_{n}=\sum_{{ }^{+} \in \mathfrak{S}_{n}}{ }^{+} D_{\alpha_{k}, \beta_{k}},{ }^{-} D_{n}=\sum_{-\mathrm{R} \in \mathfrak{S}_{n}}{ }^{-} D_{\alpha_{k}, \beta_{k}} \cdot
$$

Similarly, the potential function $V_{n}$ for the elements of $\mathrm{C}$ type, and the potential function $T_{n}$ for elements of the $\mathrm{L}$ type from the stripe $\mathfrak{S}_{n}$ will be defined as

$$
V_{n}=\sum_{\mathrm{C} \in \mathfrak{S}_{n}} V_{\alpha_{k}, \beta_{k}}, T_{n}=\sum_{\mathrm{L} \in \mathfrak{S}_{n}} T_{\alpha_{k}, \beta_{k}}
$$

Definitions (12) and (13) for the co-functions will just be completed with ${ }^{*}$ symbols. The total dissipative function $D_{n}$ and co-function $D_{n}{ }^{*}$ for the elements from the $\mathfrak{S}_{n}$ stripe will be given by the relations

$$
D_{n}={ }^{+} D_{n}-{ }^{-} D_{n}^{*}, D_{n}^{*}={ }^{+} D_{n}^{*}-{ }^{-} D_{n} .
$$

The Lagrangian $L_{n}$ and co-Lagrangian $L_{n}{ }^{*}$ for the elements from the $\mathfrak{S}_{n}$ stripe will be

$$
L_{n}=T_{n}^{*}-V_{n}, L_{n}^{*}=V_{n}^{*}-T_{n}
$$

All the definitions are conveniently arranged in Tab. 1.

With regard to definitions of the Lagrangian $L_{n}$ and the dissipative function $D_{n}$, it is obvious that the voltage at the specific element $\varepsilon_{k}$ from the $\mathfrak{S}_{n}$ stripe is

$$
v_{\varepsilon_{k}}=\left\{\begin{array}{l}
+\left(\frac{\partial D_{n}}{\partial i^{\left(\beta_{k}\right)}}\right)^{\left(-\alpha_{k}\right)} \text { for } \varepsilon_{k} \in{ }^{+} \mathrm{R} \\
-\left(\frac{\partial D_{n}}{\partial i^{\left(\beta_{k}\right)}}\right)^{\left(-\alpha_{k}\right)} \text { for } \varepsilon_{k} \in{ }^{-} \mathrm{R} \\
+\left(\frac{\partial L_{n}}{\partial i^{\left(\beta_{k}\right)}}\right)^{\left(-\alpha_{k}\right)} \text { for } \varepsilon_{k} \in \mathrm{L} \\
-\left(\frac{\partial L_{n}}{\partial i^{\left(\beta_{k}\right)}}\right)^{\left(-\alpha_{k}\right)} \text { for } \varepsilon_{k} \in \mathrm{C}
\end{array}\right.
$$

Similarly, the current through the specific element $\varepsilon_{k}$ from the $\mathfrak{S}_{n}$ stripe will be as follows:

$$
i_{\varepsilon_{k}}=\left\{\begin{array}{l}
+\left(\frac{\partial D_{n}^{*}}{\partial v^{\left(\alpha_{k}\right)}}\right)^{\left(-\beta_{k}\right)} \text { for } \varepsilon_{k} \in{ }^{+} \mathrm{R}, \\
-\left(\frac{\partial D_{n}^{*}}{\partial v^{\left(\alpha_{k}\right)}}\right)^{\left(-\beta_{k}\right)} \text { for } \varepsilon_{k} \in{ }^{-} \mathrm{R}, \\
-\left(\frac{\partial L_{n}^{*}}{\partial v^{\left(\alpha_{k}\right)}}\right)^{\left(-\beta_{k}\right)} \text { for } \varepsilon_{k} \in \mathrm{L}, \\
+\left(\frac{\partial L_{n}^{*}}{\partial v^{\left(\alpha_{k}\right)}}\right)^{\left(-\beta_{k}\right)} \text { for } \varepsilon_{k} \in \mathrm{C} .
\end{array}\right.
$$

The generalized Euler-Lagrange equation for a loop and for a node will be in the form of (17a) and (17b), respectively:

$$
\begin{aligned}
& \sum_{l}\left(\frac{\partial L_{\alpha_{l}+\beta_{l}+1}}{\partial i^{\left(\beta_{l}\right)}}\right)^{\left(-\alpha_{l}\right)}-\sum_{c}\left(\frac{\partial L_{\alpha_{c}+\beta_{c}+1}}{\partial i^{\left(\beta_{c}\right)}}\right)^{\left(-\alpha_{c}\right)}+ \\
& \sum_{r+}\left(\frac{\partial D_{\alpha_{r+}+\beta_{r+}}}{\partial i^{\left(\beta_{r+}\right)}}\right)^{\left(-\alpha_{r+}\right)}-\sum_{r-}\left(\frac{\partial D_{\alpha_{r-}+\beta_{r-}}}{\partial i^{\left(\beta_{r-}\right)}}\right)^{\left(-\alpha_{r-}\right)}=v_{\mathrm{ext}}, \\
& \sum_{c}\left(\frac{\partial L_{\alpha_{c}+\beta_{c}+1}^{*}}{\partial v^{\left(\alpha_{c}\right)}}\right)^{\left(-\beta_{c}\right)}-\sum_{l}\left(\frac{\partial L_{\alpha_{l}+\beta_{l}+1}^{*}}{\partial v^{\left(\alpha_{l}\right)}}\right)^{\left(-\beta_{l}\right)}+ \\
& \sum_{r+}\left(\frac{\partial D_{\alpha_{r+}+\beta_{r+}}^{*}}{\partial v^{\left(\alpha_{r+}\right)}}\right)^{\left(-\beta_{r+}\right)}-\sum_{r-}\left(\frac{\partial D_{\alpha_{r-}+\beta_{r-}}^{*}}{\partial v^{\left(\alpha_{r-}\right)}}\right)^{\left(-\beta_{r-}\right)}=i_{\mathrm{ext}}
\end{aligned}
$$




\begin{tabular}{|l|l|l|}
\hline Type of element & $S_{\alpha, \beta}$ & $\hat{S}_{\alpha, \beta}$ \\
\hline${ }^{+} \mathrm{R}$ & ${ }^{+} D_{\alpha, \beta}$ & ${ }^{+} D_{\alpha, \beta}^{*}$ \\
\hline $\mathrm{R}$ & ${ }^{-} D_{\alpha, \beta}^{*}$ & ${ }^{-} D_{\alpha, \beta}$ \\
\hline $\mathrm{C}$ & $V_{\alpha, \beta}$ & $V_{\alpha, \beta}^{*}$ \\
\hline $\mathrm{L}$ & $T_{\alpha, \beta}^{*}$ & $T_{\alpha, \beta}$ \\
\hline Dissip. function & $D_{n}=\sum_{\mathrm{R} \in \mathfrak{S}_{n}}{ }^{+} D_{\alpha, \beta}-\sum_{-\mathrm{R} \in \mathfrak{S}_{n}}{ }^{-} D_{\alpha, \beta}^{*}$ \\
\hline Dissip. cofunction & $D_{n}^{*}=\sum_{{ }^{+} \in \mathfrak{S}_{n}}{ }^{+} D_{\alpha, \beta}^{*}-\sum_{-\mathrm{R} \in \mathfrak{S}_{n}}{ }^{-} D_{\alpha, \beta}$ \\
\hline Lagrangian & \multicolumn{2}{|l|}{$L_{n}=\sum_{\mathrm{L} \in \mathfrak{S}_{n}} T_{\alpha, \beta}^{*}-\sum_{\mathrm{C} \in \mathfrak{S}_{n}} V_{\alpha, \beta}$} \\
\hline Co-Lagrangian & \multicolumn{2}{|l|}{$L_{n}^{*}=\sum_{\mathrm{C} \in \mathfrak{S}_{n}} V_{\alpha, \beta}^{*}-\sum_{\mathrm{L} \in \mathfrak{S}_{n}} T_{\alpha, \beta}$} \\
\hline
\end{tabular}

Tab. 1. Notation of the potential functions and co-functions, their assignment to elements in Chua's table, and the construction of $n$ th-order (co)Lagrangian and dissipative (co)function.

where $l, c, r+$ and $r$ - are the indices of the elements of types $\mathrm{L}, \mathrm{C},{ }^{+} \mathrm{R}$ and ${ }^{-} \mathrm{R}$, connected in a loop or to a node. The subscripts of the Lagrangians $L$ and the dissipative functions $D$ are equal to the number $n$ of the stripe $\mathfrak{S}_{n}$.

Equation (17a) or (17b) expresses the KVL for a loop or the KCL for a node. This equation is differential only for non-positive coefficients $\alpha_{k}$ or $\beta_{k}$. Equation for $\mathrm{KV}^{(a)} \mathrm{L}$ and $\mathrm{KC}^{(b)} \mathrm{L}$, where $a=\max \left\{\alpha_{k}\right\}, b=\max \left\{\beta_{k}\right\}$, becomes differential equation with respect to the variable $i^{\left(\beta_{\min }\right)}$ or $v^{\left(\alpha_{\min }\right)}$. The Euler-Lagrange differential equation for a loop and for a node will be in the form of (18a) and (18b), respectively:

$$
\begin{aligned}
& \sum_{l} \frac{\mathrm{d}^{a-\alpha_{l}}}{\mathrm{~d} t^{a-\alpha_{l}}}\left(\frac{\partial L_{\alpha_{l}+\beta_{l}+1}}{\partial i^{\left(\beta_{l}\right)}}\right)-\sum_{c} \frac{\mathrm{d}^{a-\alpha_{c}}}{\mathrm{~d} t^{a-\alpha_{c}}}\left(\frac{\partial L_{\alpha_{c}+\beta_{c}+1}}{\partial i^{\left(\beta_{c}\right)}}\right)+ \\
& \sum_{r+} \frac{\mathrm{d}^{a-\alpha_{r+}}}{\mathrm{d} t^{a-\alpha_{r+}}}\left(\frac{\partial D_{\alpha_{r+}+\beta_{r+}}}{\partial i^{\left(\beta_{r+}\right)}}\right)-\sum_{r-} \frac{\mathrm{d}^{a-\alpha_{r-}}}{\mathrm{d} t^{a-\alpha_{r-}}}\left(\frac{\partial D_{\alpha_{r+}+\beta_{r-}}}{\partial i^{\left(\beta_{r-}\right)}}\right)=v_{\text {ext }}^{(a)}, \\
& \sum_{c} \frac{\mathrm{d}^{b-\beta_{c}}}{\mathrm{~d} t^{b-\beta_{c}}}\left(\frac{\partial L_{c_{c}+\beta_{c}+1}^{*}}{\partial v^{\left(\alpha_{c}\right)}}\right)-\sum_{l} \frac{\mathrm{d}^{b-\beta_{l}}}{\mathrm{~d} t^{b-\beta_{l}}}\left(\frac{\partial L_{\alpha_{l}+\beta_{\beta}+1}^{*}}{\partial v^{\left(\alpha_{l}\right)}}\right)+ \\
& \sum_{r+} \frac{\mathrm{d}^{b-\beta_{r+}}}{\mathrm{d} t^{b-\beta_{r+}}}\left(\frac{\partial D_{\alpha_{r+}+\beta_{r+}}^{*}}{\partial v^{\left(\alpha_{r+}\right)}}\right)-\sum_{r-} \frac{\mathrm{d}^{b-\beta_{r-}}}{\mathrm{d} t^{b-\beta_{r-}}}\left(\frac{\partial D_{\alpha_{r-}+\beta_{r-}}^{*}}{\partial v^{\left.\alpha_{r-}\right)}}\right)=i_{\mathrm{ext}}^{(b)} .
\end{aligned}
$$

Universal equations (18a) and (18b) take specific forms for specific situations. For example, if all the elements of a loop occur in the $\mathfrak{S}_{n}$ stripe, then the Euler-Lagrange equation will be

$$
\sum_{j=0}^{b-\beta_{\min }}(-1)^{j+\beta_{\min }} \frac{\mathrm{d}^{j}}{\mathrm{~d} t^{j}}\left[\frac{\mathrm{d}}{\mathrm{d} t}\left(\frac{\partial L_{n}}{\partial i^{\left(\beta_{\min }+j\right)}}\right)+\frac{\partial D_{n}}{\partial i^{\left(\beta_{\min }+j\right)}}\right]=v_{\text {ext }}^{(a)} .
$$

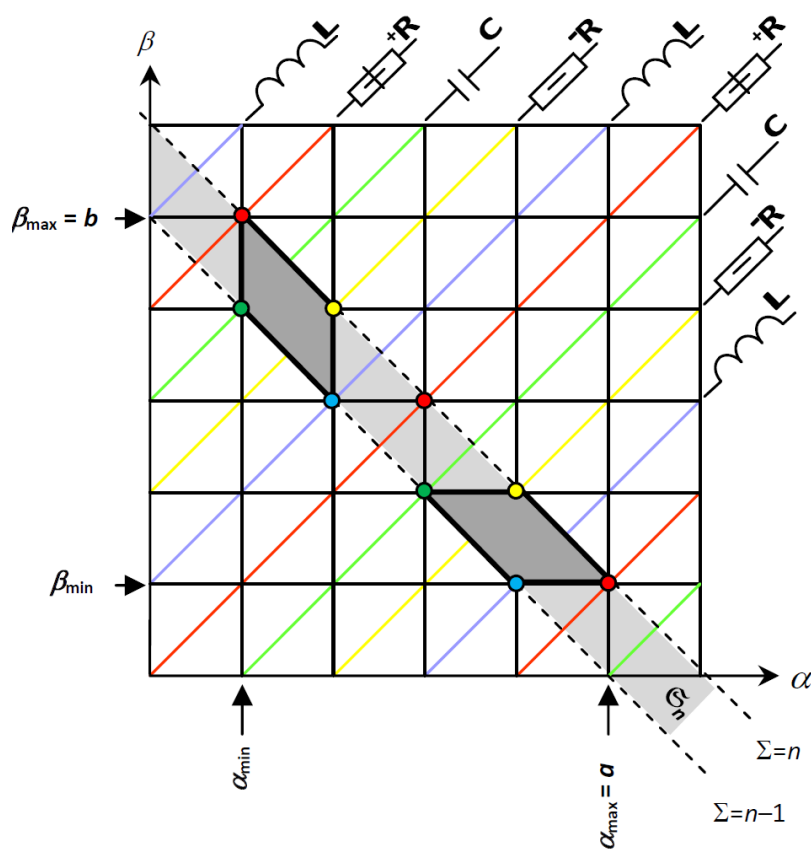

Fig. 7. An example of the case when all the elements connected in a loop or to a node lie exclusively in the $\mathfrak{S}_{n}$ stripe. Summations along the quadruples of the elements in corners of the highlighted parallelograms lead to equation (19a) or (19b).

For the elements from the $\mathfrak{S}_{n}$ stripe, connected to a node, the following equation holds:

$$
\sum_{k=0}^{a-\alpha_{\min }}(-1)^{k+\alpha_{\min }} \frac{\mathrm{d}^{k}}{\mathrm{~d} t^{k}}\left[\frac{\mathrm{d}}{\mathrm{d} t}\left(\frac{\partial L_{n}^{*}}{\partial v^{\left(\alpha_{\min }+k\right)}}\right)+\frac{\partial D_{n}^{*}}{\partial v^{\left(\alpha_{\min }+k\right)}}\right]=i_{\text {ext }}^{(b)}
$$

The layout is illustrated in Fig. 7. The summation in (19a) or (19b) is done along the quadruples of the elements of the $\left(\mathrm{L},{ }^{+} \mathrm{R}, \mathrm{C},{ }^{-} \mathrm{R}\right)$ type, which form vertices of the parallelogram

The classic form (1) of the Euler-Lagrange equation relates to the elements in the $\mathfrak{S}_{0}$ stripe, which fill three from four vertices of the above parallelograms. It is apparent that, under these conditions, Equation (19a) transforms to (1).

\section{Illustrative Example 1}

Bruton's transformation [9] is frequently used for designing active inductor-less filters from their passive RCL prototypes. It utilizes the fact that the transfer function of the filter does not change if the impedances of all elements are divided by the operator $s=\mathrm{j} \omega$. As a practical consequence, the resistors are replaced by capacitors, the inductors by resistors, and the capacitors by FDNRs (FrequencyDependent Negative Resistors) that can be synthetized using active elements. The FDNR can be modelled as an ideal $(1,-1)$ element with the respective non-linearity, possibly supplemented with additional capacitor as a result of Bruton's transformation of a resistor representing the power dissipation. 


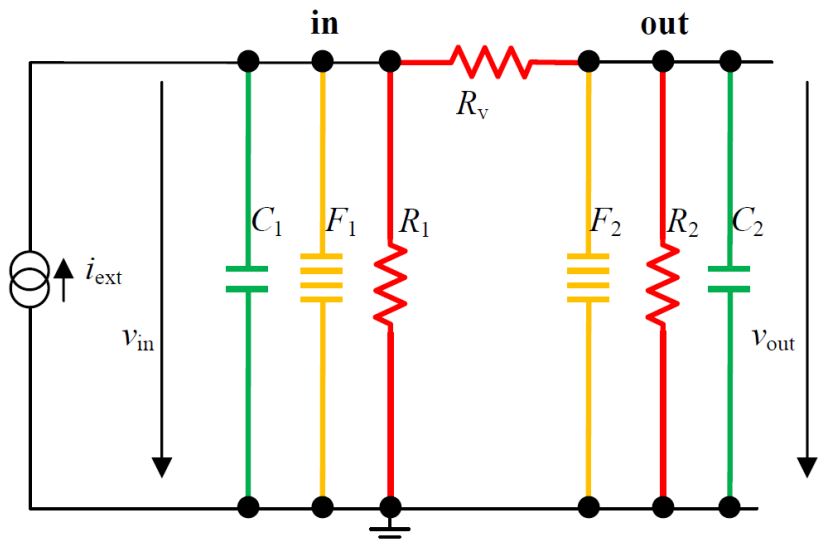

Fig. 8. Bandpass filter. Elements $F_{1}$ and $F_{2}$ are FDNRs.

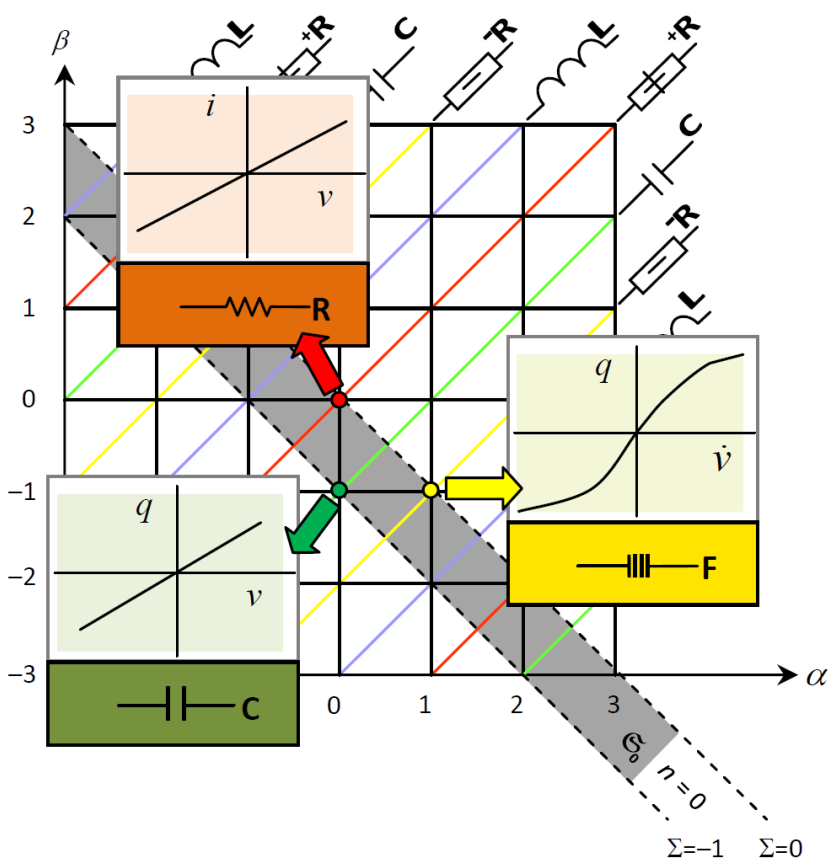

Fig. 9. Elements from the schematic diagram in Fig. 8: resistor $\mathrm{R}$, capacitor $\mathrm{C}$, and FDNR F. The dot above the voltage symbol means the differentiation with respect to time.

Figure 8 represents a bandpass filter resulting from the transformation of a passive ladder filter with mutually coupled inductors. All elements except the FDNRs are considered as linear.

As is obvious from Fig. 9, all the filter elements come from the $\mathfrak{S}_{0}$ stripe of Chua's table.

Since the filter is inductor-less, the co-Lagrangian $L_{0}{ }^{*}$ is equal to the potential co-energy of capacitors:

$$
L_{0}^{*}=V_{0}^{*}=\frac{1}{2} C_{1} v_{\text {in }}^{2}+\frac{1}{2} C_{2} v_{\text {out }}^{2} .
$$

The dissipative co-function will be

$$
\begin{array}{r}
D_{0}^{*}=\frac{1}{2} R_{1}^{-1} v_{\text {in }}^{2}+\frac{1}{2} R_{2}^{-1} v_{\text {out }}^{2}+\frac{1}{2} R_{\mathrm{v}}^{-1}\left(v_{\text {in }}-v_{\text {out }}\right)^{2}- \\
\int_{0}^{\dot{v}_{\text {in }}} q_{\mathrm{F}_{1}}(\dot{v}) \mathrm{d} \dot{v}-\int_{0}^{\dot{v}_{\text {out }}} q_{\mathrm{F}_{2}}(\dot{v}) \mathrm{d} \dot{v} .
\end{array}
$$

Since $\alpha_{\text {min }}=0, a=\alpha_{\max }=1$, and $b=\beta_{\max }=0$, the Euler-Lagrange equations are in the form

$$
\frac{\mathrm{d}}{\mathrm{d} t}\left(\frac{\partial L_{0}^{*}}{\partial \boldsymbol{v}}\right)+\frac{\partial D_{0}^{*}}{\partial \boldsymbol{v}}-\frac{\mathrm{d}}{\mathrm{d} t}\left(\frac{\partial D_{0}^{*}}{\partial \dot{\boldsymbol{v}}}\right)=\boldsymbol{i}_{\text {ext }}
$$

where $\boldsymbol{v}=\left[v_{\text {in }} v_{\text {out }}\right]^{\mathrm{T}}$ is the vector of node voltages and $\boldsymbol{i}_{\text {ext }}=\left[i_{\text {ext }} 0\right]^{\mathrm{T}}$ is the vector of current excitation. By substituting (20) and (21) into (22) we get a system of non-linear differential equations

$$
\begin{aligned}
& C_{1} \dot{v}_{\text {in }}+R_{1}^{-1} v_{\text {in }}+R_{\mathrm{v}}^{-1}\left(v_{\text {in }}-v_{\text {out }}\right)+\frac{\mathrm{d}}{\mathrm{d} t}\left(q_{\mathrm{F}_{1}}\left(\dot{v}_{\text {in }}\right)\right)=i_{\text {ext }}, \\
& C_{2} \dot{v}_{\text {out }}+R_{2}^{-1} v_{\text {out }}-R_{\mathrm{v}}^{-1}\left(v_{\text {in }}-v_{\text {out }}\right)+\frac{\mathrm{d}}{\mathrm{d} t}\left(q_{\mathrm{F}_{2}}\left(\dot{v}_{\text {out }}\right)\right)=0 .
\end{aligned}
$$

The above equations can be used for evaluating the influence of FDNR non-linearities on the filter performance.

\section{Illustrative Example 2}

The inerter, the recently discovered new mechanical element [10], is defined by unambiguous constitutive relation between force and the relative acceleration between its "free nodes". Its best-known application is connected with the mechanical absorbers of vibration and with vehicle suspension, particularly for Formula One cars [11].

Depending on the type of the applied electro-mechanical analogy, the inerter can be considered as a $(1,0)$ or $(0,1)$ element from Chua's table [12], [13]. In other words, it can be either of the $\mathrm{C}$ or $\mathrm{L}$ type but always from the stripe $\mathfrak{S}_{2}$.

The equivalent circuit in Fig. 10 starts from the absorber of vibration in [10] and transforms it to electrical form via the analogy with mass corresponding to capacitance, compliance of springs to inductance, and mechanical resistance to electrical conductance. In this type of analogy, the forces are modelled by electric currents $i$ and velocities by electric voltages $v$. The source of vibration speed is modelled by a voltage source $v_{\text {ext }}$. The inerter is expressed by the element $(\alpha, \beta)=(1,0)$ because it forms a clear bond between electric analogy of acceleration $v^{(1)}$ and electric analogy of force $i^{(0)}$.

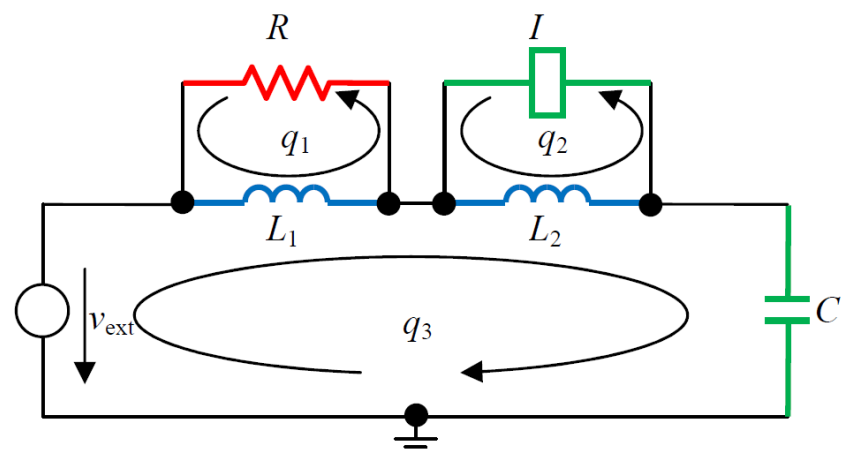

Fig. 10. Equivalent circuit of the absorber of mechanical vibration. The I element is the inerter. 


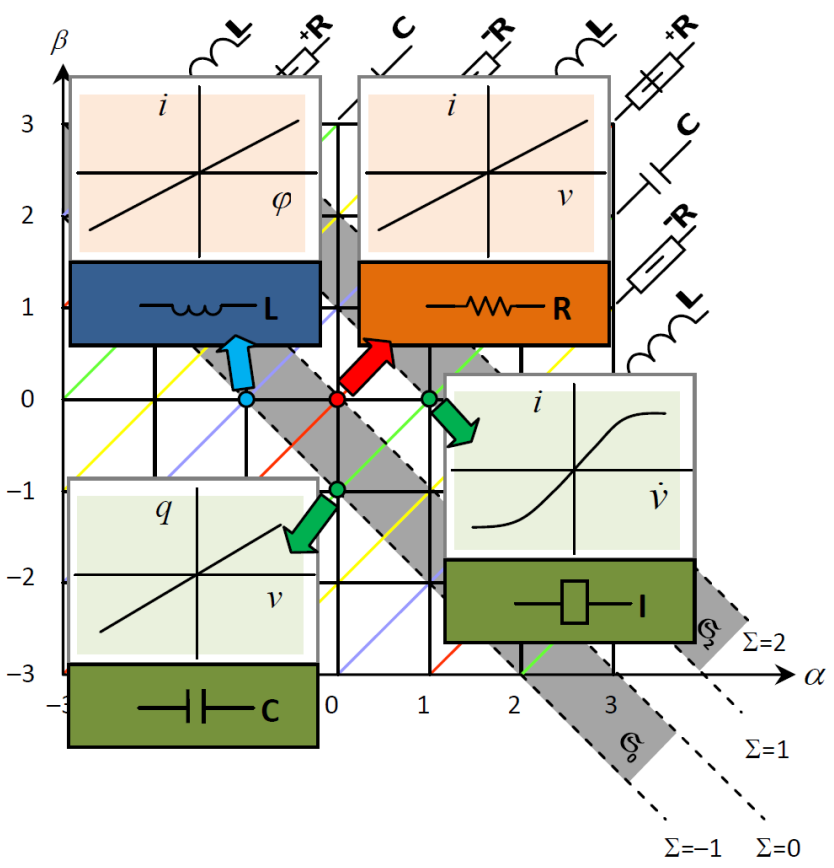

Fig. 11. Elements of the schematic diagram in Fig. 10: resistor $\mathrm{R}$, inductor $\mathrm{L}$, capacitor $\mathrm{C}$, and inerter $\mathrm{I}$. The integral of voltage $v$ with respect to time is the flux $\varphi=v^{(-1)}$.

As shown in Fig. 10, the method of the loops will be used for the circuit description. Since $\beta_{\min }=-1$ (due to the capacitor C), differential equations for charges $q=i^{(-1)}$ will be constructed via the method of loop charges.

The elements used in the schematic diagram in Fig. 10 are shown in Fig. 11. As the classical RCL elements are located in the $\mathfrak{S}_{0}$ stripe, the inerter belongs to the $\mathfrak{S}_{2}$ stripe. The corresponding Lagrangians $L_{0}$ and $L_{2}$ are

$$
\begin{gathered}
L_{0}=\frac{1}{2} L_{1}\left(\dot{q}_{1}+\dot{q}_{3}\right)^{2}+\frac{1}{2} L_{2}\left(\dot{q}_{2}+\dot{q}_{3}\right)^{2}-\frac{1}{2} C^{-1} q_{3}^{2}, \\
L_{2}=-\int_{0}^{\dot{q}_{2}} \dot{v}_{I}(\dot{q}) \mathrm{d} \dot{q} .
\end{gathered}
$$

The dissipation function $D_{0}$ is

$$
D_{0}=\frac{1}{2} R \dot{q}_{1}^{2}
$$

The Euler-Lagrange equations can be constructed according to (18a) or sequentially for stripes $\mathfrak{S}_{0}$ and $\mathfrak{S}_{2}$ according to (19a). The equation will be

$$
\frac{\mathrm{d}^{2}}{\mathrm{~d} t^{2}}\left(\frac{\partial L_{0}}{\partial \dot{\boldsymbol{q}}}\right)-\frac{\mathrm{d}}{\mathrm{d} t}\left(\frac{\partial L_{0}}{\partial \boldsymbol{q}}\right)-\frac{\partial L_{2}}{\partial \dot{\boldsymbol{q}}}+\frac{\mathrm{d}}{\mathrm{d} t}\left(\frac{\partial D_{0}}{\partial \dot{\boldsymbol{q}}}\right)=\dot{\boldsymbol{v}}_{\text {ext }}
$$

where $\boldsymbol{q}=\left[\begin{array}{ll}q_{1} & q_{2} \\ q_{3}\end{array}\right]^{\mathrm{T}}$ is the vector of loop charges and $\boldsymbol{v}_{\mathrm{ext}}=\left[\begin{array}{lll}0 & 0 & v_{\mathrm{ext}}\end{array}\right]^{\mathrm{T}}$ is the vector of voltage excitation. Substituting (24a)-(24c) in (25) yields a system of differential equations

$$
\begin{gathered}
L_{1}\left(\dddot{q}_{1}+\dddot{q}_{3}\right)+R \ddot{q}_{1}=0, \\
L_{2}\left(\dddot{q}_{2}+\dddot{q}_{3}\right)+\dot{v}_{I}\left(\dot{q}_{2}\right)=0,
\end{gathered}
$$

$$
L_{1}\left(\dddot{q}_{1}+\dddot{q}_{3}\right)+L_{2}\left(\dddot{q}_{2}+\dddot{q}_{3}\right)+C^{-1} \dot{q}_{3}=\dot{v}_{\mathrm{ext}} .
$$

In the original paper [10], the inerter is considered as a mere linear element with the constitutive relation $v^{(1)}=b^{-1} i$, where $b$ is a positive real number. A more general non-linear CR of the inerter, potentially used in (26b), can enable the analysis of the influence of such non-linearity on the operation of the vibration absorber [12].

\section{Conclusions}

The aim of the paper is to build the formalism for constructing the Euler-Lagrange equations for circuits employing arbitrary elements from Chua's table. The proposed formalism can be characterized by a generalization of the definitions of potential functions and co-functions of classical RCL elements for any $(\alpha, \beta)$ element from Chua's table, and subsequent construction of the Lagrangians and generalized dissipative functions. It should be emphasized that this procedure was selected according to the principle of Occam's razor, i.e. in the easiest possible way. The main goal was to maintain the backward compatibility with existing definitions and with the classical form of the EulerLagrange equations.

Elements whose potential functions are of the same physical dimensions naturally occur on the $\Sigma$-diagonals in Chua's table, i.e. diagonals with a constant sum $\Sigma=\alpha+\beta$. Typical examples are the contents of ${ }^{+} \mathrm{R}$ and ${ }^{-} \mathrm{R}$ for $\Sigma=0$ or energies for elements of the $\mathrm{L}$ and $\mathrm{C}$ type for $\Sigma=-1$. This fact can be used for constructing the dissipative function $D_{n}$ for every diagonal with $\Sigma=n$ ( $n$ is an odd integer), and the Lagrange function $L_{n}$ for the next diagonal with $\Sigma=n-1(n-1$ is an even integer). All elements that lie in the stripe $\mathfrak{S}_{n}$, delimited by these two diagonals, can be described by just two functions: $D_{n}$ and $L_{n}$. Today's classical form of the Euler-Lagrange equation relates to the stripe $\mathfrak{S}_{0}$. The generalized Euler-Lagrange equations can serve as recipes for obtaining equations of motion via systematic differentiation of the Lagrange and dissipative functions with respect to circuit variables and time.

The paper does not deal with the question of whether or not the extremal principle connected with the original idea of the Lagrangian is fulfilled.

\section{Acknowledgments}

The research is a part of the COST Action IC1401 and it is financially supported by the Czech Ministry of Education under grant no. LD15033. The research described in this paper was financed by the Czech Ministry of Education in frame of the National Sustainability Program under grant LO1401. For research, infrastructure of the SIX Center was used. The infrastructure of K217 UD Brno, Czech Republic, was also used. 


\section{References}

[1] JELTSEMA, D., SHERPEN, J. M. A. Multidomain modeling of nonlinear networks and systems. IEEE Control Systems, 2009, vol. 29, no. 4, p. 22-59. DOI: 10.1109/MCS.2009.932927

[2] CHUA, L. O. Memristor - The missing circuit element. IEEE Transactions on Circuit Theory, 1971, vol. 18, no. 5, p. 507-519. DOI: 10.1109/TCT.1971.1083337

[3] CHUA, L. O. Device modeling via nonlinear circuit elements. IEEE Transactions on Circuits and Systems, 1980, vol. 27, no. 11, p. 1014-1044. DOI: 10.1109/TCS.1980.1084742

[4] COHEN, G. Z., PERSHIN, Y. V., DI VENTRA, M. Lagrange formalism of memory circuit elements: classical and quantum formulations. Physical Review B, 2012, vol. 85, no. 16, p. 165428-165430. DOI: 10.1103/PhysRevB.85.165428

[5] JELTSEMA, D. Memory elements: A paradigm shift in Lagrangian modeling of electrical circuits. IFAC Proceedings Volumes, 2012, vol. 45 , no. 2 , p. 445-450. DOI: $10.3182 / 20120215-3-A T-$ 3016.00078

[6] BIOLEK, Z., BIOLEK, D., BIOLKOVÁ, V. Utilization of EulerLagrange equations in circuits with memory elements. Radioengineering, 2016, vol. 25, no. 4, p. 783-789. DOI: $10.13164 /$ re.2016.0783

[7] CHERRY, C. Some general theorems for non-linear systems possessing reactance. Philosophical Magazine, 1951, series 7, vol. 42, no. 333, p. 1161-1177. ISSN: 0031-8086. DOI: $10.1080 / 14786445108561362$

[8] MILLAR, W. Some general theorems for non-linear systems possessing resistance. Philosophical Magazine, 1951, series 7, vol. 42, no. 333, p. 1150-1160. ISSN: 0031-8086. DOI: $10.1080 / 14786445108561361$

[9] BRUTON, L. T. Network transfer functions using the concept of frequency-dependent negative resistance. IEEE Transactions on Circuit Theory, 1969, vol. 16, no. 3, p. 406-408. DOI: 10.1109/TCT.1969.1082989

[10] SMITH, M. C. Synthesis of mechanical networks: the inerter. IEEE Transactions on Automatic Control, 2002, vol. 47, no. 10, p. 1648-1662. DOI: 10.1109/TAC.2002.803532

[11] CHEN, M. Z. Q., PAPAGEORGIOU, C., SCHEIBE, F., WANG, F-C., SMITH, M. C. The missing mechanical circuit element. IEEE Circuits and Systems Magazine, 2009, vol. 9, no. 1, p. 10-26. DOI: 10.1109/MCAS.2008.931738

[12] BIOLEK, D., BIOLEK, Z., BIOLKOVÁ, V., KOLKA, Z. Nonlinear inerter in the light of Chua's table of higher-order electrical elements. In IEEE Asia Pacific Conference on Circuits and Systems. Jeju (South Korea), 2016, p. 617-620. DOI: 10.1109/APCCAS.2016.7804046

[13] BIOLEK, D., BIOLEK, Z., BIOLKOVÁ, V. Memristors and other higher-order elements in generalized through-across domain. In
IEEE International Conference on Electronics, Circuits and Systems (ICECS). Monte Carlo (Monaco), 2016, p. 604-607. DOI: 10.1109/ICECS.2016.7841274

\section{About the Authors ...}

Zdeněk BIOLEK was born in Ostrava, Czech Republic, in 1959. He received the $\mathrm{PhD}$. degree in Electronics and Informatics from Brno University of Technology, Czech Republic, in 2001.

$\mathrm{He}$ is currently with the Dept. of Microelectronics, Brno University of Technology (BUT), Czech Republic. Until the year 1993 he worked as independent researcher in the semiconductor company TESLA Rožnov. He is the author of unique electronic instruments associated with IC production and testing. He is also the author of several papers from the area of the utilization of variational principles in theoretical electrical engineering and stability testing of resistive circuits, and also from the field of memristors and mem-systems. Dr. Z. Biolek is also the co-author of two books about memristive systems and PSpice modeling and simulation of special electronic circuits including switchedcapacitor filters, switched DC-DC converters, and memristors.

Dalibor BIOLEK received the M.Sc. degree in Electrical Engineering from Brno University of Technology, Czech Republic, in 1983, and the PhD. degree in Electronics from the Military Academy Brno, Czech Republic, in 1989, focusing on algorithms of the symbolic and numerical computer analyses of electronic circuits with a view to the linear continuous-time and switched filters.

He is currently with the Dept. of EE, University of Defense Brno (UDB), and with the Dept. of Microelectronics, Brno University of Technology (BUT), Czech Republic. His scientific activity is directed to the areas of general circuit theory, frequency filters, mem-systems, and computer simulation of electronic systems. He has published over 400 papers and is the author of several books on circuit analysis and simulation. At present, he is a professor at BUT and UDB in the field of Theoretical Electrical Engineering.

Prof. Biolek is a Senior Member of the CAS/COM Czech National Group of IEEE. Currently he also serves as an Associate Editor of Electronics Letters. 